This is the author's final, peer-reviewed manuscript as accepted for publication. The publisher-formatted version may be available through the publisher's web site or your institution's library.

\title{
RNA interference revealed the roles of two carboxylesterase genes in insecticide detoxification in Locusta migratoria
}

Jianqin Zhang, Daqi Li, Pingting Ge, Meiling Yang, Yaping Guo, Kun Yan Zhu, Enbo Ma, and Jianzhen Zhang

\section{How to cite this manuscript}

If you make reference to this version of the manuscript, use the following information:

Zhang, J., Li, D., Ge, P., Yang, M., Guo, Y., Zhu, K. Y., Ma, E., \& Zhang, J. (2013). RNA interference revealed the roles of two carboxylesterase genes in insecticide detoxification in Locusta migratoria. Retrieved from http://krex.ksu.edu

\section{Published Version Information}

Citation: Zhang, J., Li, D., Ge, P., Yang, M., Guo, Y., Zhu, K. Y., Ma, E., \& Zhang, J. (2013). RNA interference revealed the roles of two carboxylesterase genes in insecticide detoxification in Locusta migratoria. Chemosphere, 93(6), 1207-1215.

Copyright: (c) 2013 Elsevier Ltd.

Digital Object Identifier (DOI): doi:10.1016/j.chemosphere.2013.06.081

Publisher's Link: http://www.sciencedirect.com/science/article/pii/S0045653513009387

This item was retrieved from the K-State Research Exchange (K-REx), the institutional repository of Kansas State University. K-REx is available at http://krex.ksu.edu 


\section{RNA interference revealed the roles of two carboxylesterase genes in insecticide detoxification in Locusta migratoria}

Jianqin Zhang ${ }^{\text {a }}$, Daqi Li ${ }^{\text {a }}$, Pingting Ge ${ }^{\text {a }}$, Meiling Yang ${ }^{\text {a }}$, Yaping Guo ${ }^{\text {a }}$, Kun Yan Zhu ${ }^{\text {b }}$, Enbo $\mathrm{Ma}^{\mathrm{a} *}$ and Jianzhen Zhang ${ }^{\mathrm{a} *}$

${ }^{a}$ Institute of Applied Biology, Shanxi University, Taiyuan, Shanxi 030006, China

${ }^{\mathrm{b}}$ Department of Entomology, 123 Waters Hall, Kansas State University, Manhattan, KS 66506, USA

Keywords: Locusta migratoria; carboxylesterase gene; insecticide detoxification; RNA interference

Running title: Roles of carboxylesterase genes in insecticide detoxification

* Corresponding authors: Tel.: +86 3517018871 or +86 351 7016098; fax: +86 3517011981 . E-mail address: maenbo2003@sxu.edu.cn (E. Ma), zjz@sxu.edu.cn (J. Zhang) 


\section{ABSTRACT}

Carboxylesterases (CarEs) play key roles in metabolism of specific hormones and detoxification of dietary and environmental xenobiotics in insects. We sequenced and characterized CarE cDNAs putatively derived from two different genes named LmCesAl and $\operatorname{LmCes} A 2$ from the migratory locust, Locusta migratoria, one of the most important agricultural pests in the world. The full-length cDNAs of LmCesA1 (1892 bp) and LmCesA2 (1643 bp) encode 543 and 501 amino acid residues, respectively. The two deduced CarEs share a characteristic $\alpha / \beta$-hydrolase structure, including a catalytic triad composed of Ser-Glu (Asp)-His and a consensus sequence GQSAG, which suggests that both CarEs are biologically active. Phylogenetic analysis grouped both LmCesA1 and LmCesA2 into clade A which has been suggested to be involved in dietary detoxification. Both transcripts were highly expressed in all the nymphal and adult stages, but only slightly expressed in eggs. Analyses of tissue-dependent expression and in situ hybridization revealed that both transcripts were primarily expressed in gastric caeca. RNA interference (RNAi) of LmCesA1 and LmCesA2 followed by a topical application of carbaryl or deltamethrin did not lead to a significantly increased mortality with either insecticide. However, RNAi of LmCesA1 and LmCesA2 increased insect mortalities by 20.9 and $14.5 \%$, respectively, when chlorpyrifos was applied. These results suggest that these genes might not play a significantly role in detoxification of carbaryl and deltamethrin but are most likely to be involved in detoxification of chlorpyrifos in L. migratoria. 


\section{Introduction}

Carboxylesterases (CarEs, EC 3.1.1.1), which are widely distributed in microbes, plants and animals, hydrolyze chemicals containing carboxylic esters to the corresponding component alcohols and acids (Satoh and Hosokawa, 2006). They share a characteristic $\alpha / \beta$-hydrolase structure, including a catalytic triad composed of Ser-Glu (Asp)-His and the nucleophilic elbow surrounding the active-site serine residue (GXSXG) (Oakeshott et al., 1999). Mammalian CarEs play an important role in biotransformation of many drugs (Imai, 2006) and transesterification of xenobiotics such as insecticides (Ahmad and Forgash, 1976). In insects, CarEs have diverse biological functions, such as metabolism of specific hormones and detoxification of dietary and environmental xenobiotics (Kontogiannatos et al., 2011). With the completions of genome sequences in various insect species, multiple CarE-encoding genes have been identified in at least eight insect species, including Drosophlia melanogaster (35 CarE genes) (Oakeshott et al., 2005), Anopheles gambiae (51) (Ranson et al., 2002), Aedes aegypti (56) (Strode et al., 2008), Apis mellifera (24) (Claudianos, 2006), Nasonia vitripennis (41) (Oakeshott et al., 2010), Tribolium castaneum (49) (Yu et. al., 2009), Bombyx mori (69) (Tsubota and Shiotsuki, 2010a), and Acyrthosiphon pisum (28) (Tsubota and Shiotsuki, 2010b).

Multiple classification schemes of CarEs existed in a wide range of organisms, including microbes, mammals and insects. Microbial CarEs have been classified into eight families (Families I to VIII) based on their conserved sequence motifs and biological properties (Arpigny and Jaeger, 1999). Mammalian CarEs have been classified into six main groups and several additional subgroups based upon the extent of amino acid homology and substrate 
selectivity (Satoh and Hoso, 2006; Holmes et al., 2009). Insect carboxyl/cholinesterases were divided into three major classes on the basis of catalytic competence and cellular/subcellular localization. By using phylogenetic analysis, insect CarEs have been partitioned into 14 major clades, denoted A to N. The oldest group (I-N) is neurodevelopmental class. The next group (D-H) is secreted catalytic class. The third and most derived group (A-C) is intracellular catalytic class with dietary detoxification functions (Oakeshott et al., 2005).

The migratory locust, Locusta migratoria (Orthoptera: Acridoidea), is one of the most important agricultural pests in many regions of the world (Chen et al., 2000a). It is widely distributed in eastern and southern Asia and the Pacific region, including Thailand, Cambodia, Indonesia, Japan, and China (Chen et al., 2000a). In recent years, the destructive outbreaks of locust had been increasing in China due to climate changes and the deteriorated ecological environment (Chen, 2000b; Xia and Huang, 2002). Three representative insecticides, including deltamethrin (pyrethroid), carbaryl (carbamate), and chlorpyrifos (organophosphate) have been used in locust control. However, frequent application of insecticides has led to the occurrence of resistance in some field populations of the locust (Ma et al., 2004; He et al., 2004; Yang et al., 2008, 2009). The major metabolic detoxification enzymes including cytochrome P450 monooxygenases (P450), esterases (particularly CarEs), and glutathione $S$-transferases (GSTs) have been found to be involved in detoxification of insecticides and development of resistance in insect populations (Ahmad et al., 1991; Yu et al., 2009; Cohen et al., 1992; Snyder et al., 1995). However, little is known about the roles of specific CarEs in detoxification of different insecticides.

In this study, we sequenced two full-length cDNAs putatively encoding different CarEs 
from L. migratoria, characterized the development- and tissue-dependent expression patterns of the two genes, and evaluated their possible roles in detoxification of three commonly used insecticides by RNA interference (RNAi) followed by bioassay. This study is expected to better understand the functional importance of locust CarEs in insecticide detoxification.

\section{Materials and methods}

\subsection{Insects}

Eggs of L. migratoria were obtained from Insect Protein Co., Ltd. (Cangzhou, China) and were hatched in a growth chamber (MGC-350NR2, Shanghai Permanent Science and Technology Co., Ltd., China) at $30 \pm 1{ }^{\circ} \mathrm{C}$ and $50 \%$ relative humidity (RH) for two weeks.

After hatching, locusts were fed on fresh wheat sprouts in plastic cages $(22 \times 22 \times 22 \mathrm{~cm})$ in the laboratory at $28 \pm 1{ }^{\circ} \mathrm{C}$ with a light: dark cycle of $14: 10 \mathrm{~h}$.

\subsection{Identification and sequencing of $c D N A s$}

The partial cDNA sequences of two locust CarE genes (EST number: LMS 002033 for $L m C e s A 1$ and LMC 003789 for LmCesA2) were searched from the locust EST database on the basis of the criteria of conserved motifs of CarEs presented in insects. These conserved motifs are SEDCLYLNI, WIHGGG, FGESAG, NKQEFGW, G-DHGDE and IPYAEPP (Satoh and Hosokawa, 1995).

The mRNA was isolated from about $800 \mu \mathrm{g}$ of total RNA from gastric caeca of locusts and $1 \mu \mathrm{g}$ of mRNA was used to synthesize the first strand of cDNA for 5'-RACE and 3'-RACE-PCR by using SMARTer ${ }^{\mathrm{TM}}$ RACE cDNA Amplification Kit following the manufacturer's instructions. Advantage ${ }^{\circledR} 2$ Polymerase (Clontech, MountainView, CA) and a 
modified PCR procedure were applied to amplify 5'-end and 3'-end cDNA fragments and the full length cDNA. The primers for RACE-PCR and full-length cDNA amplification were shown in Table 1. The PCR products from each reaction were purified using Gel Mini Purification Kit (TIANGEN, China) and subcloned into pGEM-T Easy Vector (Promega, USA) for sequencing from both directions by Beijing AuGCT Biotechnology Company. Complete sequences were analyzed by using the BLAST web tools of NCBI (http://www.ncbi.nlm.nih.gov/blast/Blast.cgi).

\subsection{Analysis of deduced amino acid sequences and phylogenetic tree}

The molecular mass (MM) and isoelectric point $(\mathrm{p} I)$ for each of the two CarEs were predicted by using ExPASy tools (http://www.expasy.ch/). The catalytic triad and substrate binding pocket for each CarE were identified with BLASTp analyses in NCBI. The potential $N$-glycosylation sites for the two CarEs were predicted by the NetNGlyc1.0 Server (http://www.cbs.dtu.dk/services/ NetNGlyc/). Signal peptide of each of the two CarEs was predicted by using SignalP4.0 program (http://www.cbs.dtu.dk/services/ signalp/).

For phylogenetic analysis, the deduced amino acid sequences of the two CarEs were aligned and converted to the meg files using MEGA5.0 software. The phylogenetic tree based on amino acid sequences of different insect CarEs was constructed by neighbor-joining method (Saitou and Nei, 1987). The bootstrap analysis of 1000 replicates was performed to evaluate branch strength. These two locust CarEs were named according to the major supported clade in which they were located and the mammalian CES nomenclature.

\subsection{Stage and tissue-dependent expression analysis}


Reverse transcription quantitative PCR (RT-qPCR) was utilized for mRNA expression analysis. For developmental expression studies, seven locust developmental stages, including egg, first-, second-, third-, fourth-, fifth-instar nymphs and adults, were collected for total RNA isolation. For tissue distribution studies, nine different tissues (brain, foregut, gastric caeca, midgut, Malpighian tubules, hindgut, fat bodies, muscles and hemolymph) were dissected from fifth-instar nynphs on day 3.

After total RNA was isolated with Trizol Plus reagent (Takara, Japan), $4 \mu \mathrm{g}$ of total RNA was used to synthesize the first strand cDNA by using First Strand cDNA Synthesis kit (Fermentas, Glen Burnie, MD) with the oligo $(\mathrm{dT})^{18}$ primer according to the manufacture's instructions. RT-qPCR was performed on ABI 7300 real-time PCR detection system (ABI, USA). Each $20 \mu$ L-reaction consisted of $10 \mu \mathrm{L}$ SYBR ${ }^{\circledR}$ Green Real-time PCR Master Mix (TOYABO, Japan), $0.4 \mu \mathrm{M}$ of each primer, $2 \mu \mathrm{L}$ of 20 -fold diluted cDNA and $6.4 \mu \mathrm{L}$ of deionized water. The RT-qPCR program consisted of $95^{\circ} \mathrm{C}$ for $1 \mathrm{~min}$, then 40 cycles of $95^{\circ} \mathrm{C}$ for $15 \mathrm{~s}, 60^{\circ} \mathrm{C}$ for $15 \mathrm{~s}$ and $72{ }^{\circ} \mathrm{C}$ for $45 \mathrm{~s}$., The melting curve was automatically added by 7300 System SDS software to detect a single gene-specific peak and check the absence of primer-dimmer peak. Relative expression was calculated using the $2^{-\Delta \Delta \mathrm{Ct}}$ method (Pfaffl, 2001) with $\beta$-actin as an internal reference gene. The RT-qPCR was repeated three times for each assay. Each replication was performed based on independent RNA sample preparation and consisted of two technical replications. The amplification efficiency of each gene was estimated from the slope given by amplification from serial 1:10 dilutions of cDNA samples according to the equation: $E=10^{[-1 / \text { slope }]}$ (Pfaffl, 2001). The investigated transcripts showed high amplification efficiency rates, 2.09 for $\operatorname{LmCes} A 1,1.94$ for $\operatorname{LmCes} A 2$ and 2.04 for $\beta$-actin $(\mathrm{n}=3)$. 
The primers used for RT-qPCR analysis were shown in Table 1.

\subsection{In situ hybridization}

To localize the transcripts of $\operatorname{LmCesA1}$ and $\operatorname{LmCes} A 2$ in gastric caeca (GC), in situ hybridization was performed. PCR products (457 bp for $L m C e s A 1$ and $361 \mathrm{bp}$ for $L m C e s A 2$ ) were first subcloned into pEASY- T3 cloning vector (TransGen, Beijing, China), and RNA sense and antisense probes were synthesized by using a T7/ Sp6 DIG RNA kit (Roche, USA). The paraffin wax-embedded gastric caeca (GC) samples were then sectioned ( $4 \mu \mathrm{m})$ and placed on Superfrost Plus slides. The tissues were dewaxed in dimethylbenzene and rehydrated in a graded series of alcohol $(100,95,80$, and $70 \%)$ and DEPC-treated water. Protein digestion was performed by incubating the samples with $20 \mu \mathrm{g} / \mathrm{ml}$ proteinase $\mathrm{K}$ (Amresco, USA) for $15 \mathrm{~min}$ at $37^{\circ} \mathrm{C}$. The sections were fixed in $10 \%$ neutral formaldehyde for 10 min, washed with PBS, incubated in $0.1 \mathrm{M}$ triethanolamine- $\mathrm{HCl}$ buffer, and treated with acetic anhydride $(0.25 \%)$ for 10 min. Finally, the tissues were prehybridized with prehybridization solution (Boster, China). Hybridization reactions were carried out at $52^{\circ} \mathrm{C}$ overnight with hybridization mix (Boster, China) containing an appropriate concentration of digoxigenin-labeled RNA probe in the hybridization oven. After hybridization, the slides were washed in $4 \times$ standard sodium citrate (SSC, once), $2 \times \mathrm{SSC}$ (twice), $1 \times \mathrm{SSC}$ (twice) and $0.2 \times$ SSC (once) at $42^{\circ} \mathrm{C}$. The tissues were then blocked with $1 \%$ blocking reagent (Roche) in Buffer I (100 mM Tris, $150 \mathrm{mM} \mathrm{NaCl}$; $\mathrm{pH} 7.5)$ for $2 \mathrm{~h}$ at $42^{\circ} \mathrm{C}$ and incubated with anti-digoxigenin-AP Fab fragments (Roche, 1:1000 dilution) for $2 \mathrm{~h}$ at room temperature. After 30 min of washing in Buffer I, the tissues were visualized with NBT-BCIP and mounted on slides. The pictures were acquired using Olympus BX51 microscope equipped with 
Olympus U-DPT-2 CCD and ImagePro software. The integrity of the GC sections was confirmed by staining with hematoxylin and eosin and reviewed by standard light microscopy.

\subsection{RNAi to evaluate the roles of two CarE genes in insecticide detoxification}

Our earlier studies demonstrated that $L m C e s A 1$ and $L m C e s A 2$ (previously named as $L m C a r E 9$ and $L m C a r E 25$, respectively) were involved in malathion detoxification in $L$. migratoria (Zhang et. al., 2011). To examine possible involvement of these genes in detoxification of other commonly used insecticides (chlorpyrifos, carbaryl and deltamethrin), we performed insecticide bioassays with each of the three insecticides after each of the two genes was silenced by RNAi. The gene-specific primers and the lengths of the PCR products that were used for double-stranded RNA (dsRNA) syntheses for these two genes are shown in Table 1. The dsRNA were prepared as previously described by Zhang et al. (2011). Briefly, after PCR products were purified, they were used as templates for dsRNA syntheses using T7 RiboMAX ${ }^{\mathrm{TM}}$ Express RNAi System (Promega, USA) according to manufacturer's instructions. The synthesized dsRNA samples were dissolved in deionized water, and each dsRNA was adjusted to a final concentration of $1.5 \mu \mathrm{g} \cdot \mu \mathrm{L}^{-1}$. RNAi experiments were conducted by injecting $2 \mu \mathrm{L}$ of $L m C e s A 1$ or $L m C e s A 2$ dsRNA (treatment) or $2 \mu \mathrm{L}$ of dsGFP (control) into the abdomen between the second and third abdominal segments of each second-instar nymph (2-day old) by using a microsyringe.

To examine silencing efficiency of each gene at different time points, 60 nymphs were used for mRNA expression analysis at 12, 24 and $48 \mathrm{~h}$ after dsRNA injection. Nine nymphs from control or each gene-specific dsRNA treated group were randomly divided into three subgroups and each subgroup was used as a biological sample for total RNA isolation by using 
Trizol Plus reagent (Takara, China). The first strand cDNA was synthesized from $4 \mu \mathrm{g}$ total RNA using First Strand cDNA Synthesis kit (Fermentas), and $2 \mu \mathrm{L}$ of 20 -fold diluted cDNA was used for RT-qPCR based on the same method as described in Section 2.4.

In order to examine any possible increases in insect susceptibility to each insecticide after RNAi, we chose a relatively low dose (approximately $\mathrm{LD}_{30}$ ) of each insecticide in our bioassay. This low dose will allow a sufficient increase in insect mortality of the insects in response to the insecticide treatment if the target CarE gene that is silenced by RNAi plays an important role in detoxification of the test insecticide. For each insecticide bioassay after RNAi, 39 nymphs from each treated group (injected with $L m C e s A 1$ or $L m C e s A 2$ dsRNA) or control group (injected with GFP dsRNA) at $24 \mathrm{~h}$ were divided into three subgroups as replications, and a droplet of $3 \mu \mathrm{L}$ of acetone containing $60 \mathrm{ng}$ carbaryl, $15 \mathrm{ng}$ chlorpyrifos or $0.6 \mathrm{ng}$ deltamethrin was topically applied onto the abdomen between the second and third sterna of each nymph. These insecticide doses were predetermined to be approximately $\mathrm{LD}_{30}$ by bioassay. Mortality was recorded at $24 \mathrm{~h}$ after locusts exposed to the insecticide. Nymphs were considered dead if they were not able to walk in a coordinative way when touched with a brush.

\section{Results}

\subsection{Identification and classification of two CarE genes}

The full length cDNA sequences of two CarE genes were obtained by 3'-end and 5'-end RACE-PCR using the cDNA template prepared from the insect gastric caecum. Phylogenetic tree was constructed by using neighbor-joining method to investigate the evolutionary relationships among the two locust CarE and other insect CarE proteins (Fig. 2). The two 
locust CarEs firstly grouped together and then clustered into clade A. Based on the nomenclature of mammalian CarEs (Holmes et al., 2008), the two L. migratoria CarE genes

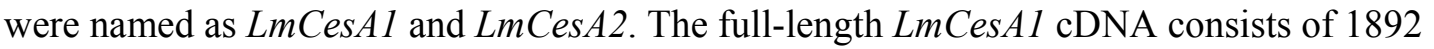
nucleotides, including an open reading frame (ORF) of 1629 nucleotides encoding 543 amino acid residues, and 54 and 209-nucleotide non-translated regions at 5' and 3' ends, respectively (Fig. 1). The complete cDNA of $\operatorname{LmCesA2}$ consists of 1643 nucleotides, including an ORF of 1503 nucleotides encoding 501 amino acid residues, and 39 and 101- nucleotide noncoding regions at 5' and 3' ends, respectively. The nucleotide accession numbers are JX961710 for LmCesA1 and JX961711 for LmCesA2 in GenBank

Compared with other insect CarE amino acid sequences found in GenBank using BLAST-P at NCBI website, the LmCesA1 shares 39\% identity with Megachile rotundata esterase E4-like (XP_003700585), whereas LmCesA2 shares 38\% identity with Bombyx mori alpha-esterase 45 (NP_001104822). Both LmCesA1 and LmCesA2 display conserved motifs, including CarE superfamily specific catalytic triad and substrate binding pocket. The triads are Ser205-Glu338-His453 in LmCesA1and Ser159-Glu291-His400 in LmCesA2. The multiple noncontiguous residues, GGG126-128QSA204-206 A209M365ST374-375T407G454A457 for LmCesA1 and GGG78-80QSA158-160 M163A313PT321-322G355S401L404 for LmCesA2, may form their substrate binding pockets (Table1). The calculated molecular mass and $\mathrm{p} I$ are $59.4 \mathrm{kDa}$ and 4.85 for LmCesA1, and $55.8 \mathrm{kDa}$ and 4.37 for LmCesA2, respectively, by using the ExPASy Proteomics website. SignalP4.0 analysis predicted that both LmCesA1 and LmCesA2 have a signal peptide at their N-terminus (Fig. 1). Potential $N$-glycosylation sites were observed at Asn261-Asn262-Thr263, Asn262-Thr263-Ser264 and 
Asn523-Leu524-Ser525 in LmCesA1 and Asn392-Val393-Ser394 in LmCesA2 (Fig. 1).

\subsection{Tissue and stage-dependent expression patterns of CarE genes in L. migratoria}

Tissue-dependent expression patterns of $L m C e s A 1$ and $L m C e s A 2$ were analyzed by RT-qPCR in nine tissues, including brain, foregut, gastric caeca, midgut, Malpighian tubules, hindgut, fat bodies, muscles and hemolymph. As shown in Fig. 3, both LmCesA1 and $L m C e s A 2$ were highly expressed in intestine. However, $L m C e s A 1$ was predominantly expressed in gastric caeca and midgut, whereas $\operatorname{LmCes} A 2$ was mainly expressed in gastric caeca, with only slight expressions in other tissues.

The stage-dependent expression patterns of $\operatorname{LmCes} A 1$ and $\operatorname{LmCes} A 2$ were also examined in eggs, five different nymphal instars (first-, second-, third-, fourth- and fifth), and adults by RT-qPCR. LmCesA1 was highly expressed in all the nymphal and adult stages, with slight expression in eggs. However, $\operatorname{LmCes} A 2$ was highly expressed in first- and second-instar nymphs, but relatively low in other developmental stages examined.

\subsection{Localization of LmCesA1 and LmCesA2 transcripts in gastric caecum}

The cellular localizations of $L m C e s A 1$ and $L m C e s A 2$ transcripts in gastric caecum of $L$. migratoria were examined by in situ hybridization. As shown in Fig. 4, both LmCesAl and LmCesA2 transcripts were detected in the basal of the columnar cells of gastric caecum (Fig. 4B and Fig. 4C).

\subsection{Effect of gene silencing on locust susceptibility to insecticides}

RT-qPCR analyses at different times after the injection of dsRNA for each target gene showed significantly decreased transcript levels of LmCesA1 at $24 \mathrm{~h}$ (about 87\%) and $48 \mathrm{~h}$ 
(about 86\%) and of LmCesA2 at $24 \mathrm{~h}$ (about 97\%) as compared with those in the controls, indicating an effective silencing of these two genes at $24 \mathrm{~h}$ after dsRNA injection (Fig. 5A, Fig. $5 B)$.

To investigate possible involvements of $\operatorname{LmCes} A 1$ and $L m C e s A 2$ in detoxification of three commonly used insecticides (carbaryl, chlorpyrifos and deltamethrin), the susceptibility of the dsRNA-injected locusts to each of these insecticides was assessed. Results from our insecticide bioassays showed that the susceptibility of the nymphs to chlorpyrifos increased from 34.2 to $55.1 \%$ when $L m C e s A 1$ was silenced, and from 34.2 to $48.7 \%$ when $L m C e s A 2$ was silenced (Fig. 5C). In contrast, the susceptibility of the nymphs to deltamethrin decreased from 33.3 to $19.3 \%$ after $L m C e s A 1$ was silenced and from 33.3 to $18.5 \%$ after $L m C e s A 2$ was silenced (Fig. 5C). However, the susceptibility of the insects to carbaryl did not change significantly when $\operatorname{LmCesA1}$ or $\operatorname{LmCesA2}$ was silenced.

\section{Discussion}

In our previous studies, we identified 25 cDNA fragments putatively derived from different CarE genes from locust EST database, and arbitrally named them from LmCarE1 to LmCarE25 because it was difficult to provide systematic nomenclature only based on their cDNA fragments (Zhang et al., 2011). In this study, we obtained the full-length cDNAs of two locust CarE genes and evaluated their phylogenetic relationships with other known insect CarEs. Based on the nomenclature of mammalian CarEs (Holmes et al., 2008), we named these two locust CarE genes as LmCesA1 and LmCesA2.

The predicted amino acid sequences of the two CarE genes were found to be highly similar to the known insect CarEs and displayed conserved motifs for substrate binding pocket 
and catalytic triad (Cygler et al., 1993) when analyzed by BLASTp with credible expectation values (E-value $\leq 4 \mathrm{e}^{-119}$ for LmCesA1, E-value $\leq 5 \mathrm{e}^{-100}$ for LmCesA2). The predicted signal peptide cleavage sites of their proteins were found using the SignalP4.0 Server. Thus, both CarEs might be secreted enzymes. The potential $N$-glycosylation sites for mammalian CarEs have been observed at one or several positions (Kroetz et al., 1993; Fleming et al., 2005; Holmes et al., 2010). Interestingly, these sites were also found in the two locust CarEs. It has been reported that the role of the $\mathrm{N}$-glycosylated carbohydrate group is to maintain CarE stability and catalytic efficiency (Kroetz et al., 1993). Such a property is likely to be shared by both mammalian and insect CarEs. This is particularly true for LmCesA1 that contains two such sites for $N$-glycosylation.

Different tissue distributions of CarE family members may imply the diverse roles of CarE family members in both mammalians and insects (Pindel et al., 1997; Xu et al., 2002; Holmes et al., 2010; Yu et al., 2009). Indeed, several studies have demonstrated that midgut is the first barrier of xenobiotics in peroral toxicity (Ahmad et al., 1991; Yu et al., 2009; Cohen et al., 1992; Snyder et al., 1995). It has been reported that gastric caeca are supplementary structures of the midgut for increasing the surface area for food absorption, water uptake and waste expulsion (Akpan and Okorie, 2003). Similarly, our investigation of the expression patterns of two locust CarE genes in nine tissues showed that both LmCesA1 and LmCesA2 were highly expressed in gastric caeca, whereas $\operatorname{LmCesA1}$ was also expressed relatively higher in the midgut than in other tissues except for gastric caeca. Therefore, we propose that these two CarEs may play important roles in detoxification of xenobiotics including insecticides that are ingested by the insect. Stage-dependent expression patterns of two locust CarE genes were 
determined in all developmental stages by RT-qPCR. Both genes were highly transcribed across all the feeding stages including five nymphal instars and adult stage when the insect is likely to come in contact with xenobiotics. The lowest expression levels of both genes were found in eggs, particularly for $L m C e s A 1$ whose expression level was undetectable by RT-qPCR. Our results support our notion that these two genes may contribute to the detoxification of xenobiotics derived from their food.

Organophosphates (OPs), carbamates and pyrethroids are three major classes of insecticides used for pest control. CarEs are important in the hydrolysis of a broad range of endogenous and xenobiotic ester-containing compounds, such as OPs, carbmates, and pyrethroids. Extensive application of these insecticides resulted in the development of resistance against these insecticides in many insect species. In many cases, insecticide resistance is mediated by CarEs with the molecular mechanisms of gene overexpression (e.g., gene amplification) and mutations of the genes (Li et al., 2007). In Myzus persicae, the overexpression of a CarE (E4) gene by gene amplification contribute to the enhanced degradation and sequestration of many insecticides including OPs, carbamates and pyrethroids (Field and Devonshire, 1998). Previous studies have demonstrated that the increased CarE activity is responsible for malathion resistance in L. migratoria (Yang et al., 2008b). Although carbamates and pyrethroids have been used for locust control, little is known about detoxification of these insecticides by CarEs in the locust. Nevertheless, it is relatively difficult to pinpoint the detoxification roles of individual CarE genes since several dozens of CarE genes have been identified in each insect species (Ranson et al., 2002; Oakeshott et al., 2005; Strode et al., 2008). 
We took the advantage of using RNAi to evaluate possible detoxification roles of $\operatorname{LmCes} A 1$ and $\operatorname{LmCesA2}$ in locusts. If silencing of a particular gene by RNAi significantly increases the susceptibility of the insect to an insecticide, it is possible that this gene may play a role in detoxification of this insecticide because silencing of the gene can ultimately diminish the detoxification of the insecticide by the enzyme encoded by the gene. Base on this principle, our previous studies have demonstrated that many genes encoding detoxification enzymes, including $L m G S T$ and $L m C Y P$, play key roles in the detoxification of different insecticides (Qin et. al., 2012; Guo et. al., 2012). To evaluate whether or not LmCesA1 and $\operatorname{LmCes} A 2$ played a role in detoxifying the three representative insecticides (carbaryl, chlorpyrifos and deltamethrin) in the locust, we performed RNAi for $\operatorname{LmCesA1}$ and $\operatorname{LmCes} A 2$ followed by bioassay with each of the three insecticides. Our results suggested that both genes play an important role in detoxification of chlorpyrifos in the locust. Thus, it is reasonable to predict that the increased expression of LmCesA1 and LmCesA2 in L. migratoria might lead to the elevated tolerance to chlorpyrifos or other xenobiotics, which may consequently result in difficulties in controlling the locust in the field.

However, silencing $\operatorname{LmCes} A 1$ or $\operatorname{LmCes} A 2$ showed significantly lower mortality when the locusts were exposed to deltamethrin as compared with the controls (dsGFP injection). We proposed that these two genes did not play a role in deltamethrin detoxification. However, we don't know how silencing $\operatorname{LmCesA1}$ or $\operatorname{LmCesA2}$ can reduce the susceptibility of $L$. migratoria. A possible explanation is that $\operatorname{LmCesA1}$ and $\operatorname{LmCes} A 2$ may also play an important role in insect physiology and silencing these genes by RNAi may modify the insect response to deltamethrin. Further studies are needed to clarify this outcome of RNAi. Nevertheless, our 
results support the notion that both $\operatorname{LmCesA1}$ and $\operatorname{LmCes} A 2$ play a significant role in chlorpyrifos detoxification as validated by RNAi followed by insecticide bioassay. Our present studies may help us better understand functions of the insect CarE genes and provide a roadmap to use RNAi techniques to evaluate possible functions of detoxification genes in insects. 


\section{Acknowledgments}

This work was supported by National Natural Science Foundation of China (Grant Nos. 30810103907, 31172161, 31071980), Research Fund for the Doctoral Program of Higher Education of China (Grant Nos. 20111401110006, 20101401120008), Natural Science Foundation of Shanxi Province of China (Grant No. 2011011033-1) and Public Welfare Fund for Agriculture (Grant No. 200903021). The authors thank Prof. Le Kang (Institute of Zoology, Chinese Academy of Sciences) for sharing the resource of the locust EST database platform. 


\section{References}

Ahmad, S., Duval, D.L., Weinhold, L.C., Pardini, R.S., 1991. Cabbage looper antioxidant enzymes: tissue specificity. Insect Biochem. 215, 563-572.

Ahmad, S., Forgash, A.J., 1976. Nonoxidative enzymes in the metabolism of insecticides. Drug Metab. Rev. 13, 141-164.

Akpan, B.E. Okorie, T.G., 2003. Allometric growth and performance of the gastric caeca of Zonocerus variegatus (L.) (Orthoptera: Pyrgomorphidae). Acta Entomol. Sinica 46, 558-566.

Arpigny, J.L., and Jaeger K.E., 1999. Bacterial lipolytic enzymes: classification and properties. Biochem. J. 343:177-183.

Chen, Y.L., 2000a. Main achievement of research and management of migratory locust in China. Entomol. Knowl. 37, 50-55.

Chen, Y.L., 2000b. The characteristic, cause and ecological management of locust disaster. Bulletin Biol. 35, 1-5.

Claudianos, C., Ranson, H., Johnson, R.M., Biswas, S., Schuler, M.A., Berenbaum, M.R., Feyereisen, R., Oakeshott, J.G., 2006. A deficit of detoxification enzymes: Pesticide sensitivity and environmental response in the honeybee. Insect Mol. Biol. 15, 615-636.

Cohen, M.B., Schuler, M.A., Berenbaum, M.R., 1992. A host-inducible cytochrome P450 from a host-specific caterpillar: Molecular cloning and evolution. Proc. Natl. Acad. Sci. USA 89, 10920-10924.

Cygler, M., Schrag, J.D., Sussman, J.L., Harel, M., Silman, I., Gentry, M.K., Dostor, 
B.P., 1993. Relationship between sequence conservation and three-dimensional structure in a large family of esterases, lipases and related proteins. Protein Sci. $2,366-382$.

Field, L.M., Devonshire, A.L., 1998. Evidence that the E4 and FE4 esterase genes responsible for insecticide resistance in the aphid Myzus persicae (Sulzer) are part of a gene family. Biochem. J. 330, 169-73.

Fleming, C.D., Bencharit, S., Edwards, C.C., Hyatt, J.L., Tsurkan, L., Bai, F., Fraga, C., Morton, C.L., Howard-Williams, E.L., Potter, P.M., Redinbo, M.R., 2005. Structural insights into drug processing by human carboxylesterase 1: tamoxifen, mevaststin, and inhibition by Benzil. J. Mol. Biol. 352, 165-177.

Guo, Y.Q., Zhang, J.Z., Yu R.R, Zhu, K.Y., Guo, Y.P., Ma, E.B., 2012. Identification of two new cytochrome P450 genes and RNA interference to evaluate their roles in detoxification of commonly used insecticides in Locusta migratoria. Chemosphere 87, 709-717.

He, Y.P., Ma, E.B., Zhu, K.Y., 2004. Characterizations of general esterases in relation to malathion susceptibility in two field populations of the oriental migratory locust, Locusta migratoria manilensis (Meyen). Pestic. Biochem. Physiol. 78, $103-113$.

Holmes, R.S., Cox, L.A., VandeBerg, J.L., 2008. Mammalian carboxylesterase 5: comparative biochemistry and genomics. Comp Biochem Physiol. D 3, 195-204.

Holmes, R.S., Cox, L.A., VandeBerg, J.L., 2009. A new class of mammalian carboxylesterase CES6. Comp. Biochem. Physiol. Part D Genomics Proteomics 


\section{4, 209-217.}

Holmes, R.S., Cox, L.A., VandeBerg, J.L., 2010. Mammalian carboxylesterase 3: comparative genomics and proteomics. Genetica. 138, 695-708.

Imai, T., 2006. Human carboxylesterase isozymes: catalytic properties and rational drug design. Drug Metab. Pharmacok. 21, 173-185.

Jagadeshwaran, U., Vijayan, V.A., 2009. Biochemical characterization of deltamethrin resistance in a laboratory-selected strain of Aedes aegypti. Parasitol. Res. 104, $1431-1438$.

Kontogiannatos, D., Michail, X., Kourti, A., 2011. Molecular characterization of an ecdysteroid inducible carboxylesterases with GQSCG motif in the corn borer, Sesamia nonagrioides. J. Insect Physiol. 57, 1000-1009.

Kroetz, D.L., McBride, O.W., Gonzalez, F.J., 1993. Glycosylation-dependent activity of Baculovirus-expressed human liver carboxylesterases: cDNA cloning and characterization of two highly similar enzyme forms. Biochem. 32, $11606-11617$.

Li, X.C., Schuler, M.A., Berenbaum, M.R., 2007. Molecular mechanisms of metabolic resistance to synthetic and natural xenobiotics. Annu. Rev. Entomol. 52, 231-253.

Ma, E.B., He, Y.P., Zhu, K.Y., 2004. Comparative studies of acetylcholinesterases purified from two field populations of the oriental migratory locust (Locusta migratoria manilensis): Implications of insecticide resistance. Pestic. Biochem. Physiol. 78, 67-77.

Oakeshott, J.G., Claudianos, C., Campbell, P.M., Newcomb, R.D. and Russell, R.J., 
2005. Biochemical genetics and genomics of insect esterases, in Comprehensive molecular Insect Science. Vol. 5, ed. by Gilbert, L.I., Iatrou, K. and Gill, S. Elsevier, Oxford, UK, pp. 309-381.

Oakeshott, J.G., Claudianos, C., Russell, R.J., Robin, G.C., 1999. Carboxyl/cholinesterases: a case study of evolution of successful multigene family. Bioessays 21, 1031-1042.

Oakeshott, J.G., Johnson, R.M., Berenbaum, M.R., Ranson, H., Cristino, A.S., Claudianos, C., 2010. Metabolic enzymes associated with xenobiotic and chemosensory responses in Nasonia vitripennis. Insect Mol. Biol. 19 (Suppl. 1), 147-163.

Pfaffl, M.W., 2001. A new mathematical model for relative quantification in real-time RT-PCR. Nucleic Acids Res. 29, 2002-2007.

Pindel, E.V., Kedishvili, N.Y., Abraham, T.L., Brezinski, M.R., Zhang, A., Dean, R.A., Bosron, W.F., 1997. Purification and cloning of a broad substrate specificity human liver carboxylesterase that catalyzes the hydrolysis of cocaine and heroin. J. Biol. Chem. 272, $14769-14775$.

Qin, G.H., Jia, M., Liu T., Zhang, X.Y., Guo, Y.P., Zhu, K.Y., Ma, E.B., Zhang, J.Z., 2012. Heterologous expression and characterization of a sigma glutathione S-transferase involved in carbaryl detoxification from oriental migratory locust, Locusta migratoria manilensis (Meyen). J. Insect Physiol. 58, 220-227.

Ranson, H., Claudianos, C., Ortelli, F., Abgrall, C., Emingway, J., Sharaknova, M. V., Unger, M.F., Collins F.H., Feyereisen, R., 2002. Evolution of supergene families associated with insecticide resistance. Science 298, 179-181. 
Saitou, N, and Nei, M., 1987. The neighbour-joining method: a new method for reconstructing phylogenetic trees. Mol. Biol. Evol. 4, 406-425.

Satoh, T. Hosokawa, M., 1995. Molecular aspects of carboxylesterase isoforms in comparison with other esterases. Toxicol. Let. 82-83, 439-445.

Satoh, T., Hosokawa, M., 2006. Structure, function and regulation of carboxylesterase. Chem. Biol. Interact. 162, 195-211.

Snyder, M.J., Stevens, J.L., Andersen, J.F. and Feyereisen, R., 1995. Expression of cytochrome P450 genes of the CYP4 family in midgut and fat body of the tobacco hornworm. Manduca sexta. Arch. Biochem. Biophysi. 321, 13-20.

Soderlund, D.M., Knipple, D.C., 2003. The molecular biology of knockdown resistance to pyrethroid insecticides. Insect Biochem. Mol. Biol. 33, 563-577.

Strode, C., Wondji, C.S., David, J.-P., Hawkes, N.J., Lumjuan, N., Nelson, D.R., Drane, D.R., Karunaratne, S.H.P.P., Hemingway, J., Black Iv, W.C., Ranson, H., 2008. Genomic analysis of detoxification genes in the mosquito Aedes aegypti. Insect Biochem. Mol. Biol. 38, 113-123.

Tsubota, T. and Shiotsuki, T., 2010a. Research article Genomic analysis of carboxyl/cholinesterase genes in the silkworm Bombyx mori. BMC Genomics 11, 377.

Tsubota, T. and Shiotsuki, T., 2010b. Genomic and phylogenetic analysis of insect carboxyl/cholinesterase genes. J. Pestic. Sci. 35, 310-314.

Xia, J.Y., Huang, H., 2002. Analysis on the outbreak of Locusta migratoria manilensis and its control strategies. Plant Protect Tech. Ext. 22, 7-10. 
Xu, G., Zhang, W., Ma, M.K., MacLeod, H.L., 2002. Human carboxylesterase 2 is commonly expressed in tumor tissue and is correlated with the activation of irinotecan. Clin. Cancer Res. 8, 2605-2611.

Yang, M.L., Zhang, J.Z., Zhu, K.Y., Xuan, T., Liu, X.J., Guo, Y.P., Ma, E.B., 2008a. Increased activity and reduced sensitivity of acetylcholinesterase associated with malathion resistance in a field population of the oriental migratory locust, Locusta migratoria manilensis (Meyen). Pestic. Biochem. Physiol. 91, 32-38.

Yang, M.L., Zhang, J.Z., Zhu, K.Y., Xuan, T., Liu, X.J., Guo, Y.P., Ma, E.B., 2008 b. Mechanisms of organophosphate resistance in a field population of oriental migratory locust, Locusta migratoria manilensis (Meyen). Arch. Biochem. Physiol. $69,1-10$.

Yu, Q.Y., Lu, C., Li, W.L., Xiang, Z.H., Zhang, Z., 2009. Annotation and expression of carboxylesterases in the silkworm, Bombyx mori. BMC Genomics 10, 553.

Zhang, J.Z., Zhang, J.Q., Yang, M.L., Jia, Q.D., Guo, Y.P., Ma, E.B., Zhu, K.Y., 2011. Genomics-based approaches to screening carboxylesterase genes potentially involved in malathion resistance in oriental migratory locust (Locusta migratoria manilensis). Pest Manag. Sci. 67, 183-190. 
Table 1. Primers used for full-length cDNA sequencing, qRT-PCR analysis and dsRNA synthesis of $\operatorname{LmCes} A 1$ and $\operatorname{LmCesA2}$

\begin{tabular}{|c|c|c|c|}
\hline Gene & Application & Primer sequences (5'-3') & Product size (bp) \\
\hline \multirow[t]{12}{*}{ LmCesA1 } & RT-PCR & F: CAGAACCTCCTGTTGGAACACA & 77 \\
\hline & & R: CAGAGCATCTCTTACACCATTCCAT & \\
\hline & 5'-RACE & R: CATGTTGTCCAGTGATATGAGGCAC & 102 \\
\hline & 3'-RACE & F: GAATGGGATTTGGTCCTGACTACTT & 1440 \\
\hline & Full length amplication & F: ACGCGGGGTAAGGAAGAAAGT & 1842 \\
\hline & & R: AGTGTAGTCATGGAACAGAACGTG & \\
\hline & In situ hybridyzation & F: CTTCTGTCTCAGGGGGCTTAC & 457 \\
\hline & & R: GTTGCAGATTCTCACCACCTC & \\
\hline & dsRNA & $\mathrm{F}:$ TAATACGACTCACTATAGGG & 311 \\
\hline & & AAAGTGCTGGTGGTGCTTCT & \\
\hline & & R: TAATACGACTCACTATAGGG & \\
\hline & & ATGCTGTGGCTCAACACTTG & \\
\hline \multirow[t]{12}{*}{$\operatorname{LmCes} A 2$} & RT-PCR & F: CTCATCTCCTACGGCGTCATC & 74 \\
\hline & & R: TCACCAGTCGTGAGGAAACCT & \\
\hline & 5'-RACE & R: TCGGTATCGGAGCCGCTGTTGTAGT & 443 \\
\hline & 3'-RACE & F: ACGGACGATTCGCAACAGTTGGTAG & 968 \\
\hline & Full length amplication & F: GACAACATTGACCATGGAACT & 1536 \\
\hline & & R: AAGGTGTCAGTCTTATTTTACAC & \\
\hline & In situ hybridyzation & F: AACAGCAGCAGGAGGCG & 361 \\
\hline & & R: GTAGGGTTTCCGTATTTAGCG & \\
\hline & dsRNA & F: TAATACGACTCACTATAGGG & 331 \\
\hline & & CGGGTGGAATGTCTGTATCC & \\
\hline & & R: TAATACGACTCACTATAGGGG & \\
\hline & & ATTGGAGACTCCGGCATTA & \\
\hline \multirow[t]{4}{*}{ GFP } & dsGFP & F: TAATACGACTCACTATAGGG & 712 \\
\hline & & GTGGAGAGGGTGAAGG & \\
\hline & & R: TAATACGACTCACTATAGGG & \\
\hline & & GGGCAGATTGTGTGGAC & \\
\hline \multirow[t]{2}{*}{$\beta$-actin } & RT-PCR & F: CGAAGCACAGTCAAAGAGAGGTA & 156 \\
\hline & & R: GCTTCAGTCAAGAGAACAGGATG & \\
\hline
\end{tabular}




\section{Figure Legends:}

Fig. 1. Nucleotide and deduced amino acid sequences of the two CarE cDNAs from $L$.

migratoria. Red letters denote the catalytic triad. Letters in grey shadow represent substrate binding pocket. The stop codon at the end of the ORF is highlighted in bold. Putative signal peptides predicted by the SignalP4.0 program (http://www.cbs.dtu.dk/services/SignalP/) are shown in green. Potential $N$-glycosylation sites are highlighted in blue. The sequences were deposited in the GenBank (Accession No.: JX961710 (A) and JX961711(B)).

Fig. 2. An unrooted neighbor-joining tree showing phylogenetic relationships of selected insect CarEs. The tree was generated by MEGA 5 based on amino acid sequences. Nodes with $>50 \%$ bootstrap values (1000 replicates) are indicated. The nomenclatures of the clades are based on Oakeshott et al. (2005). Blue triangles indicate Anopheles gambiae CarEs, purple squares Drosophila melanogaster CarEs, red rhombus Locusta migratoria CarEs, black circles other insects CarEs (including AAL09822 Aphis gossypii, CAA88030 Culex quinquefasciatus, AF153367 Choristoneura fumiferana, P12992 Heliothis virescens, AF287267 Bombyx mori, AF327882 Manduca sexta). The GenBank accession numbers for various insect CarEs used in this tree were generally taken from the National Center for Biotechnology Information (NCBI) database (http://www,ncbi.nl.nih.gov/Genbank/). The five clades were named as A, C, G, H and $\mathrm{N}$.

Fig. 3. Tissue- and stage-dependent expression patterns of $L m C e s A 1$ and $L m C e s A 2$ in $L$.

migratoria. A, Nine tissues were selected to examine the mRNA expresion levels of two CarE 
genes. Brain (BR), foregut (FG), gastric caeca (GC), midgut (MG), hindgut (HG), Malpighian tubules (MT), fatbodies (FB), muscles (MU) and hemolymph (HE). B, Stage-dependent expression patterns of two CarE genes. Tissue- and Stage-dependent expressions of two genes were evaluated by qRT-PCR. $\beta$-actin was used as an internal reference gene. Vertical bars indicate standard errors of the mean $(n=3)$. Different letters on the bars indicate that the means are significantly different among tissues or different stages in Fisher's LSD multiple comparison test $(P<0.05)$.

Fig. 4. In situ hyhybridizition of $\operatorname{LmCes} A 1$ and $\operatorname{LmCes} A 2$ on gastric caeca (GC) sections of $L$. migratoria. A) paraffin-embedded thin section of gastric caecum showing the overall structure and corresponding regions where the transcripts were detected as shown in Panels B and C. B) LmCesA1 transcript was detected in the basal membrane of columnar cell of gastric caeca shown by purple color. C) LmCesA2 transcript was detected in the basal membrane of columnar cell of gastric caeca shown by purple color. $\mathrm{B}^{\prime}$ ) and $\mathrm{C}^{\prime}$ ) represent the enlarged regions with abundant $\operatorname{LmCesA1}$ and $\operatorname{LmCesA2}$ transcripts, respectively. Cc, Columnar cell; Bm, basal membrane; Cm, Ciliated membrane.

Fig. 5. Susceptibility of L. migratoria to each of three selected insecticides after LmCesA1 and $\operatorname{LmCes} A 2$ were silenced. A, B: Silencing efficiency of $L m C e s A 1$ and $L m C e s A 2$ after the locust nymphs were injected with $3 \mu \mathrm{g}$ dsRNA (ds $G F P$ as control). RNA was extracted and quantified by qRT-PCR at 12, 24 and $48 \mathrm{~h}$ after the injection. $\beta$-actin was used as an internal reference gene. A: LmCesA1; B: LmCesA2; Vertical bars indicated standard errors of the mean 
$(n=3)$. Different letters on the bars indicate that the means are significantly different among controls in Fisher's LSD multiple comparison test $(P<0.05)$. Significant differences were statistically separated by $t$-test $(* P<0.05, * * P<0.01)$. (C) Susceptibility of the locusts to different insecticides after dsRNA injection in second-instar nymphs. Insecticides bioassays were conducted by topical application at $24 \mathrm{~h}$ after dsRNA injection. Mortality of the locusts was assessed at $24 \mathrm{~h}$ after each insecticide treatment. Averaged data from three independent experiments were analyzed together with S.E. Asterisks on the bars indicate that the means are significantly different among the control and treatments (student's t-test, $* \mathrm{P}<0.05, * * \mathrm{P}<$ $0.01)$. 
Fig. 1.

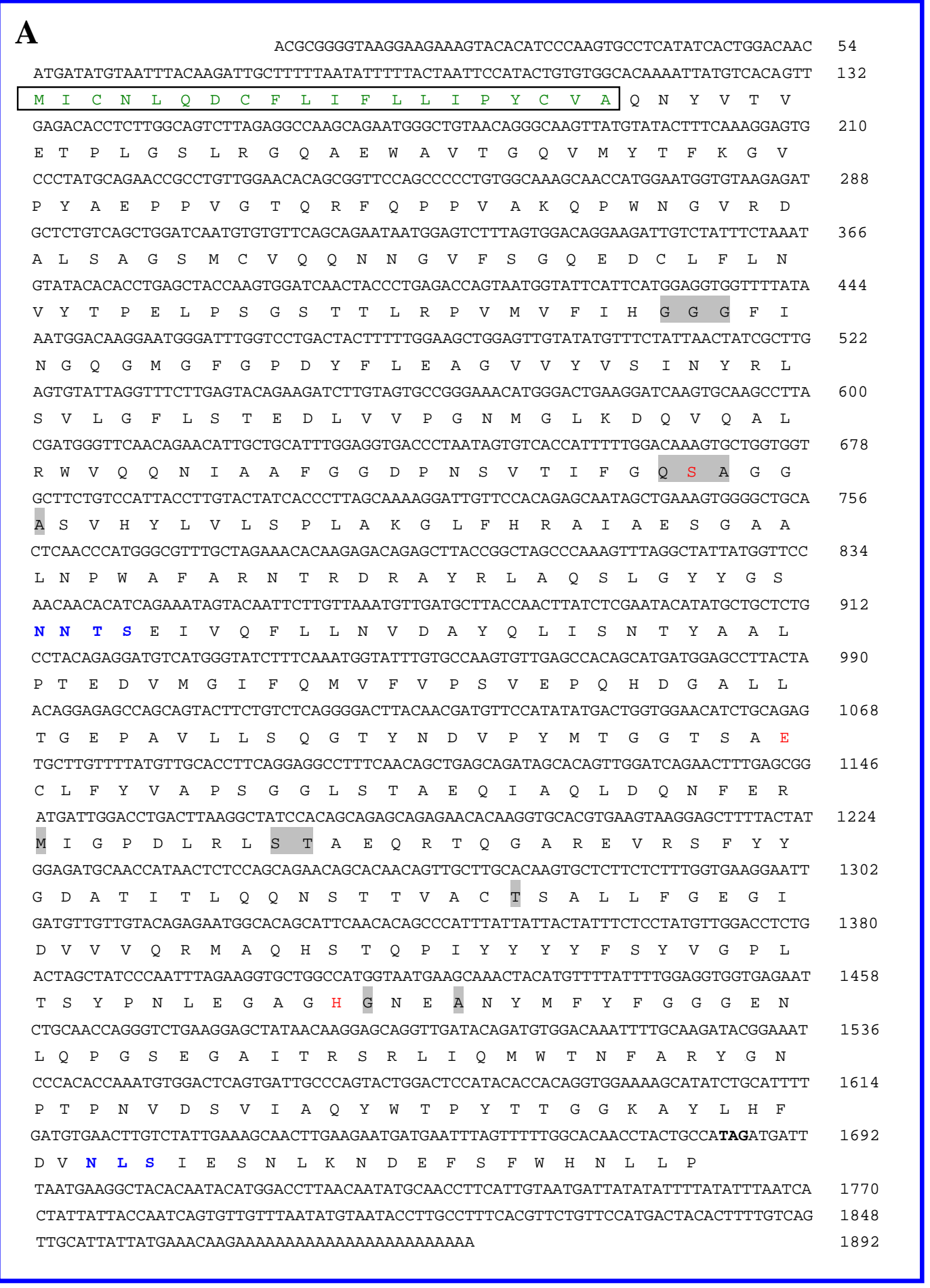




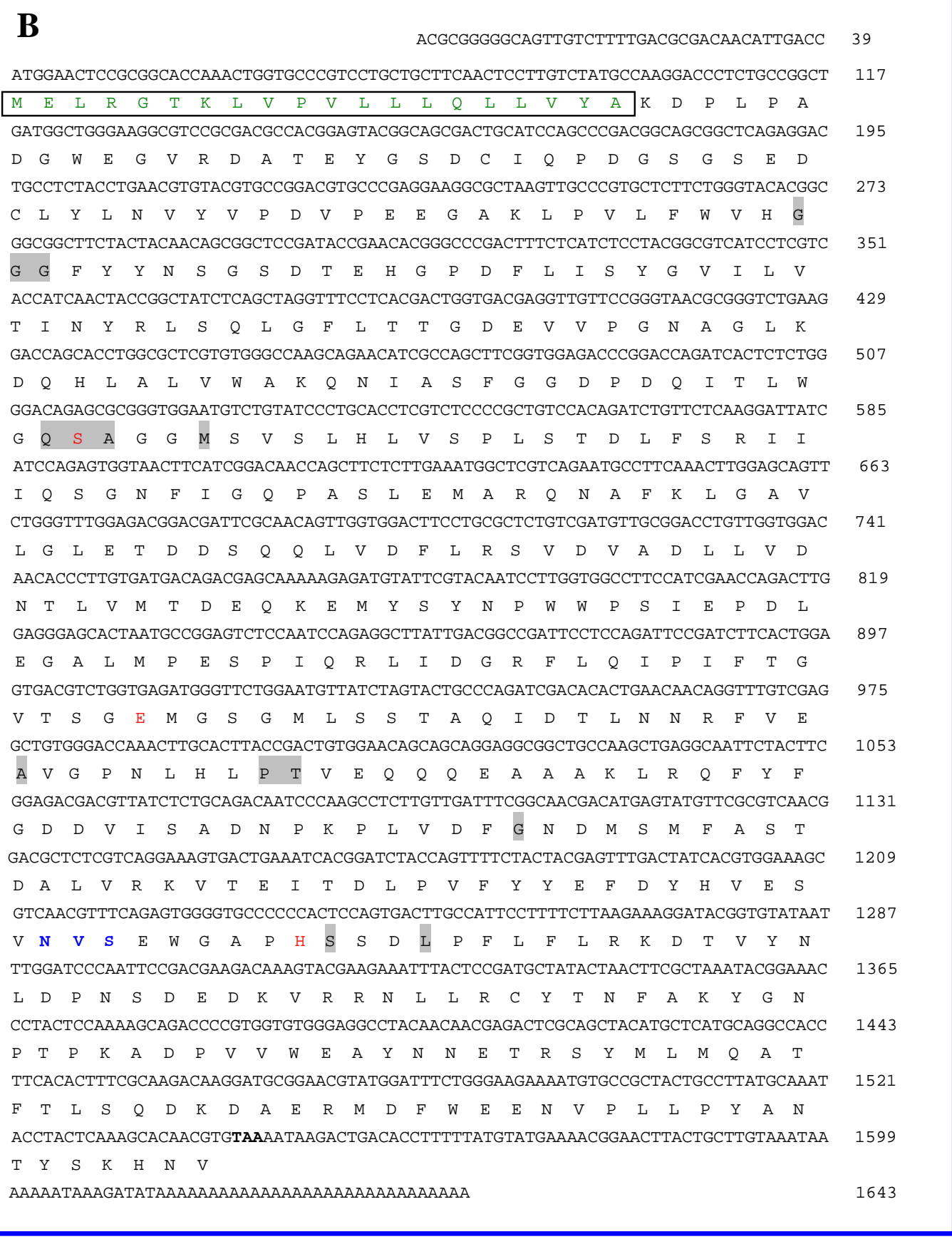


Fig. 2.

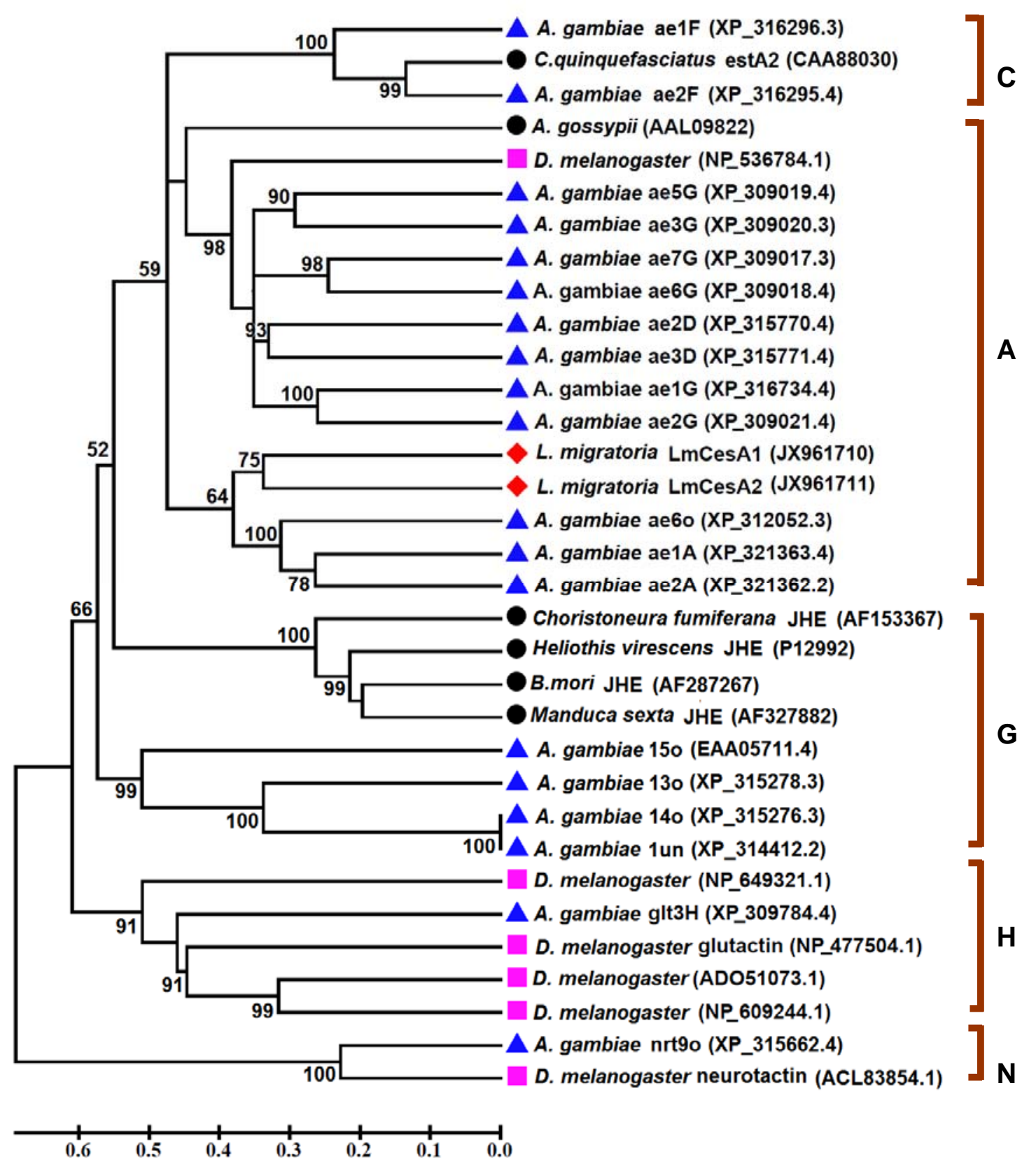


Fig. 3.
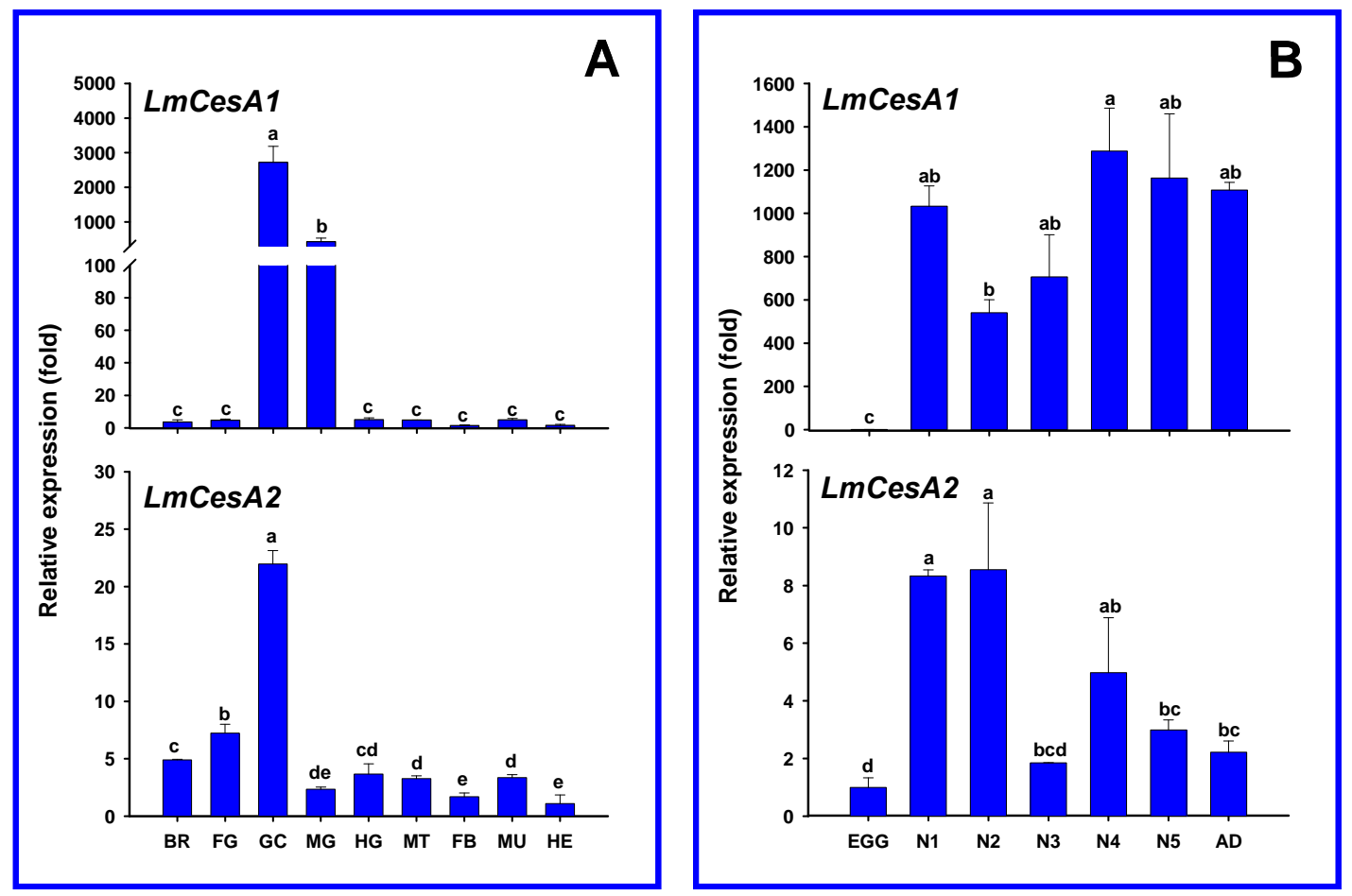
Fig. 4.

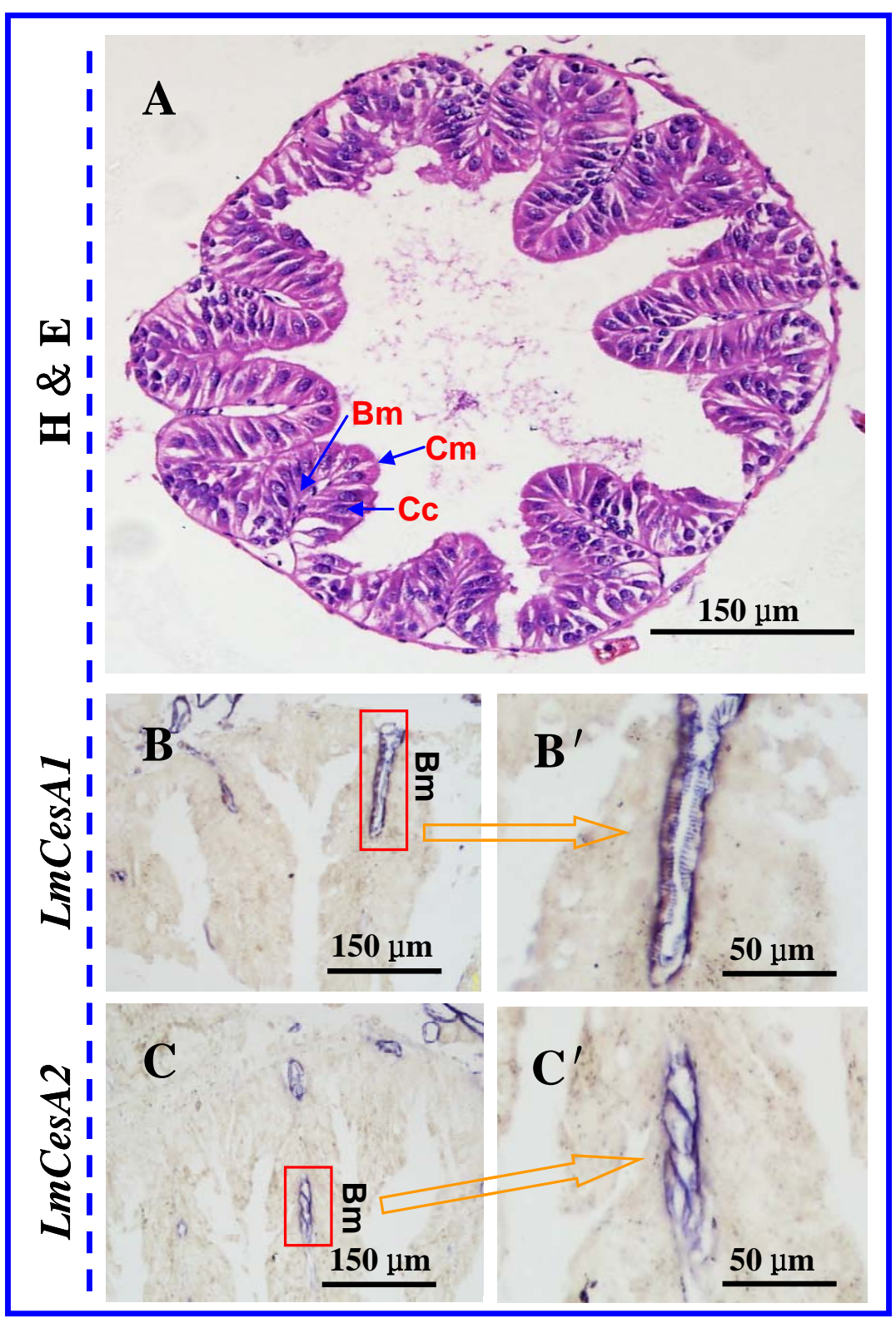


Fig. 5.
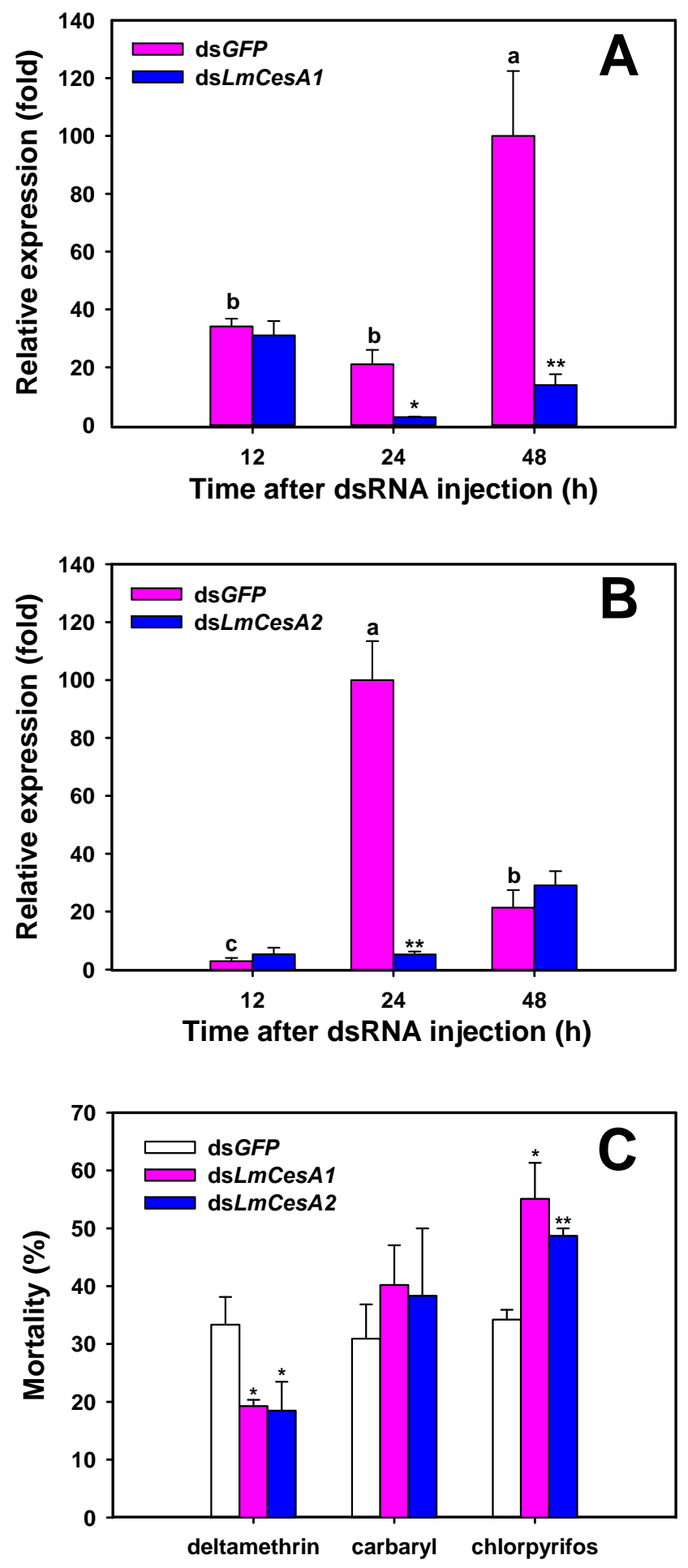